\title{
Dual Role of Metallic Lithium in the Initiation of Vinyl Polymerization
}

\author{
V.R. PAI VERNEKER* and B. SHAHA, Department of Inorganic and Physical \\ Chemistry, Indian institute of Science, Bangalore 560 012, India
}

\section{INTRODUCTION}

Alkali-metal-initiated polymerization of dienes has been known since the $1910 \mathrm{~s}^{1,2}$ Metallic sodium and potassium have been known as ionic initiators for quite some time. ${ }^{3}$ However, the designation of metallic lithium as an initiator has not always been unequivocal. Among the various alkali metals lithium occupies a unique position in the periodic table in respect of its marked differences in reactivity with other members of the same group. This dissimilarity betewen lithium and other alkali metals seems to prevail during their initiation of polymerization reactions also; e.g., Stavely et al. ${ }^{4}$ observe lithium metal to be unique as an initiator in the polymerization of isoprene in that it strongly favors the formation of cis-1,4 structure (with a trace of 3,4) to the exclusion of trans-1,4 and -1,2 structures, while metals such as sodium, potassium, cesium, and rubidium give primarily a mixture of cis and trans structures. Tobolsky et al. ${ }^{5-7}$ similarly observed in their study of the copolymerization of styrene and methyl methacrylate that lithium differs from sodium in reactivity. They further observed that their data were not sufficient to designate lithium as either classically ionic or a typical free-radical initiator. ${ }^{6}$ However, Overberger and Yamamoto ${ }^{8}$ from an ${ }^{1} \mathrm{H}$ NMR investigation of the same system concluded the absence of radical propagation and vouched for the existence of radical anions at least in the beginning of polymerization, as was originally proposed by Szwarc. ${ }^{9}$

In order to ascertain the behavior of metallic lithium as an initiator for polymerization, we have carried out homopolymerization of acrylonitrile and acrylamide with lithium metal as initiator utilizing temperature as the only varient. The problem may be tentatively worked out according to the following scheme:

If metallic lithium behaves differently from other alkali metals, viz., sodium or potassium, during polymerization of a vinyl monomer, e.g., acrylonitrile (AN), then in all probability the initiating species would be different in the two cases. Assuming sodium metal to be a typical anionic initiator, as is generally believed, ${ }^{3}$ the initiating species of AN with sodium metal as initiator would always be the same, viz., anion radical under all possible variable conditions. However, if lithium metal could behave as a free radical as well as an ionic initiator as Tobolsky et al. speculated, ${ }^{5-7}$ then under suitable conditions different initiating species viz., (a) free radical, and (b) anion radical would be present in a system consisting of AN with metallic lithium as initiator. Now, if one chooses a suitable device to detect such initiating species, it would be a relatively simple matter to trace the differences between the two initiator types. The case in question lends itself to study by ESR spectroscopy.

The identification of different initiating species in the metallic-lithium-initiated homopolymerization of acrylamide (AM) is relatively simple - though indirect. It

\footnotetext{
*Present address: Martin Marietta Laboratories, 1450 South Rolling Rd., Baltimore, MD 21227.
} 
is well known that $A M$ undergoes vinyl polymerization via 1,2 addition to give polyacrylamide (PAM) when heated alone or with a free-radical initiator. However, AM undergoes hydrogen transfer polymerization via 1,4 addition to give an entirely different polymer - poly $(\beta$-alanine) (PBAL) - when treated with an anionic initiator. ${ }^{10}$ PAM may be distinguished from PBAL in a number of ways, viz., PBAL gives characteristic peptide and imide bands in IR spectra around 1540 and 3080 $\mathrm{cm}^{-1}$, respectively, ${ }^{11,12}$ which PAM lacks; both PAM and PBAL may be hydrolyzed and the hydrolyzed product subjected to ninhydrin test - a negative response would unequivocally confirm the presence of PAM. However, the most promising easy identification of these two polymers, even when present in a mixture, would be through NMR spectroscopy. It is known that PAM gives two groups of peaks in ${ }^{1} \mathrm{H}$ NMR spectra centered around $1.67 \delta$ and $2.22 \delta$ corresponding to the methylene and methine protons, respectively, with integral ratio 2:1.13,14 $\mathrm{PBAL}$ also gives two groups of peaks in ${ }^{1} \mathrm{H}$ NMR spectra but at positions significantly different from those of PAM, viz., at $2.48 \delta$ and $3.42 \delta$ corresponding to the methylene protons attached to $>\mathrm{C}=\mathrm{O}$ and $-\mathrm{NH}$ - groups, respectively, with integral ratio 1:1.14,15 If lithium behaves differently under different conditions, then one would be able to identify the products - PAM or PBAL or both - from a cursory look at its ${ }^{1} \mathrm{H}$ NMR spectra and hence would be able to trace its reaction course.

\section{EXPERIMENTAL}

$\mathrm{AN}^{16}$ and $\mathrm{AM}^{17}$ were purified according to established procedures. ESR spectra were taken with a Varian E-line century spectrometer operating at a microwave frequency of $9.05 \mathrm{GHz}$. ESR spectra were taken after keeping AN with the initiator at the desired temperature(s) in the absence of oxygen and moisture $\left(1 \times 10^{-2} \mathrm{~mm}\right.$ $\mathrm{Hg}$ ) for a specific period and recording the spectra at $-142^{\circ} \mathrm{C} .{ }^{1} \mathrm{H}$ NMR spectra of PAM and PBAL were taken with a Bruker model WH 270-MHz spectrometer in $\mathrm{D}_{2} \mathrm{O}$ (ca. $0.5 \%$ solution) at room temperature with TSP as internal standard. UV spectra of polyacrylonitrile (PAN) were recorded with a Beckman model 25 spectrophotometer in dimethylformamide. X-ray diffraction pattern of PAN samples were taken with a Philips model PW 1050/70 vertical goniometer with nickel-filtered $\mathrm{Cu}$ Koc radiation.

\section{RESULTS}

\section{ESR Studies}

ESR spectra of metallic-lithium- and sodium-initiator acrylonitrile systems are shown in Figure 1. An analysis of Figures 1(a) and 1(b) reveals that the shape of the ESR signal of sodium-initiated AN systems remains the same at both temperatures $\left(-33,+65^{\circ} \mathrm{C}\right)$ studied though the magnitude of the spectroscopic splitting factor $g$ increases from 2.00041 at $-33^{\circ} \mathrm{C}$ to 2.00304 at $+65^{\circ} \mathrm{C}$ with increase in temperature. However, the shape of the ESR signal of lithium-initiated AN systems is found to be different at the two temperatures studied [Figs. 1(c,d)]. The low-temperature $\left(-33^{\circ} \mathrm{C}\right)$ one-line signal is found to give rise to a strong one-line signal with a shoulder in the high-field region at high temperature $\left(+65^{\circ} \mathrm{C}\right)$. The $g$ value of the signal at $-33^{\circ} \mathrm{C}$ and the shoulder component of the signal at $+65^{\circ} \mathrm{C}$ of the lithium-initiated AN system is found to be very close to that of DPPH, while the strong signal at $+65^{\circ} \mathrm{C}$ is found to have $g$ value slightly greater than that of DPPH. This is suggestive of the fact that whereas there is only one type of initiating species with sodium metal as the initiator for $\mathrm{AN}$, in the lithium-initiated $\mathrm{AN}$ system there are two 


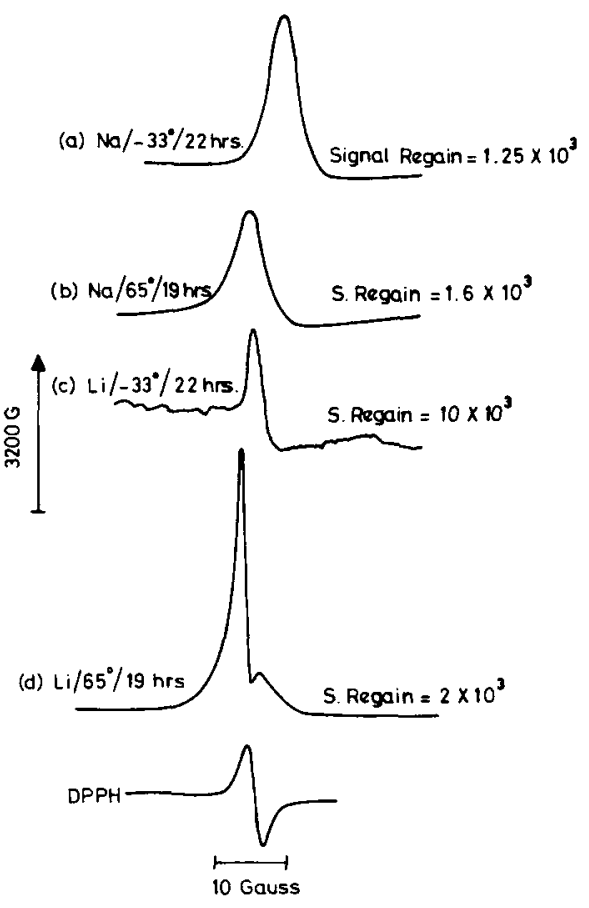

Fig. 1. ESR spectra of sodium-initiated AN (a,b), lithium-initiated AN (c,d) recorded at $-142^{\circ} \mathrm{C}$.

different initiating species depending on the temperature of the system. In both cases, however, the $g$ value is found to increase slightly with increase in temperature. It may be noted that so far ESR signal with AN system has been obtained with either free-radical initiator ${ }^{18}$ or with energetic radiations. ${ }^{19}$ This is the first time so far as we know that ESR signals have been obtained for AN system with a metal initiator.

Further, it has been observed that lithium-initiated polymerization of AN at $-33^{\circ} \mathrm{C}$ is completely suppressed with hydroquinone (no polymer in $720 \mathrm{~h}$ ) - the same recipe, however, could be polymerized at $+65^{\circ} \mathrm{C}$ to a colored polymer in about $98 \mathrm{~h}$ (yield $30 \%$ ). However, sodium-initiated polymerization of $\mathrm{AN}$ at $-33^{\circ} \mathrm{C}$ was found to go on uninterruptedly in the presence of hydroquinone. This is indicative of the fact that lithium-initiated polymerization of $\mathrm{AN}$ at $-33^{\circ} \mathrm{C}$ is free-radical in nature, while that with sodium metal under identical conditions is ionic. This contention finds support from the fact that the UV, ${ }^{1} \mathrm{H} \mathrm{NMR},{ }^{13} \mathrm{C} \mathrm{NMR}$, and x-ray diffraction patterns of lithium-initiated polymer obtained at $-33^{\circ} \mathrm{C}$ are identical to those of a typical free-radical (AIBN)-initiated polymer obtained from $\mathrm{AN}$ at $+65^{\circ} \mathrm{C}$.

\section{${ }^{1} \mathrm{H}$ NMR Studies}

The ${ }^{1} H$ NMR spectrum of a typical PAM obtained from AM with a free-radical initiator is shown in Figure 2(a) and that of a typical PBAL obtained from AM with metallic lithium initiator (at $100^{\circ} \mathrm{C}, 1 \times 10^{-2} \mathrm{~mm} \mathrm{Hg}$ ) is shown in Figure $2\left(\mathrm{~b}\right.$ )..$^{14}$ The ${ }^{1} \mathrm{H}$ NMR spectrum of the product obtained from AM by initiation with lithium metal from dioxan at $19 \pm 1^{\circ} \mathrm{C}\left(1 \times 10^{-2} \mathrm{~mm} \mathrm{Hg}\right)$ is shown in Figure 2(c). An examination of Figures 2(a)-2(c) reveals that both PAM and PBAL are present in 


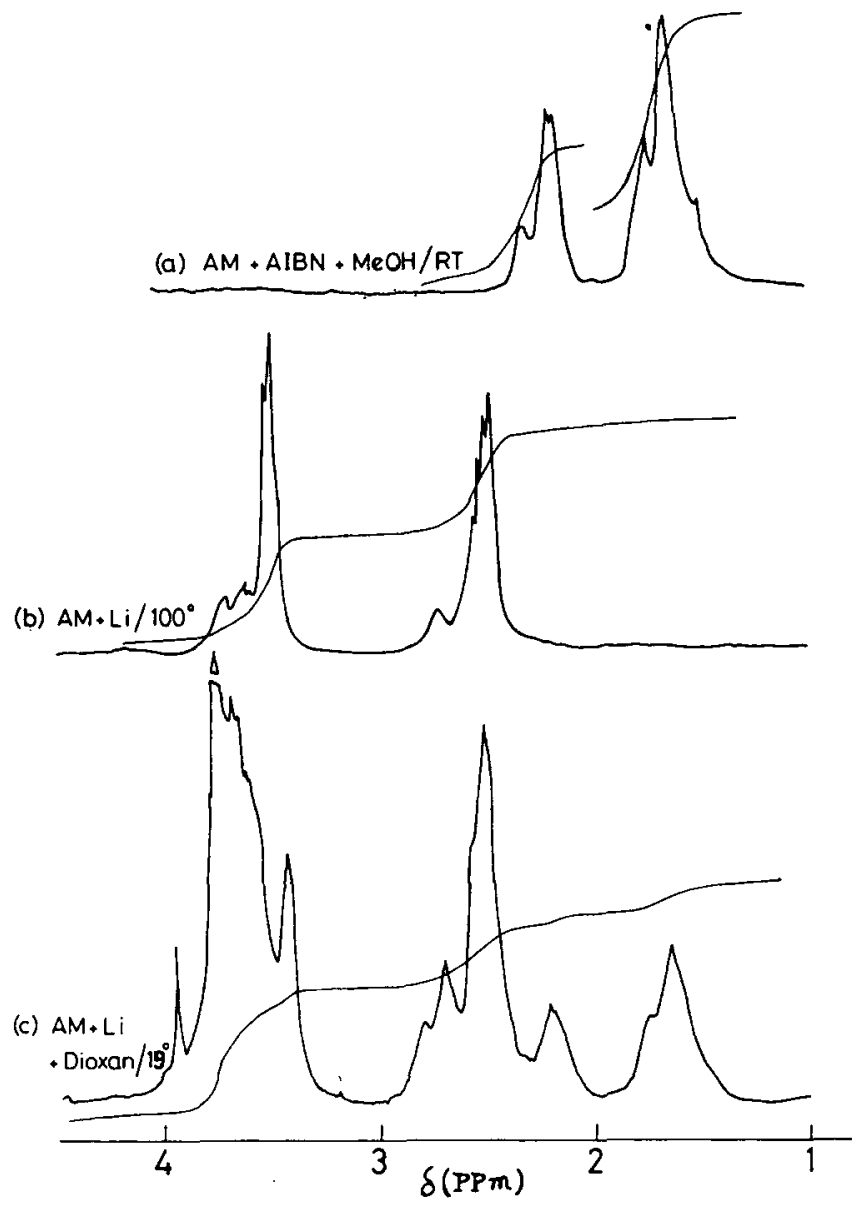

Fig. 2. ${ }^{1} \mathrm{H}$ NMR spectra of (a) AIBN-initiated PAM, (b) Li-initiated PBAL at $100^{\circ} \mathrm{C}$, (c) Liinitiated product from $A M$ in Dioxan at $19^{\circ} \mathrm{C}$.

the product obtained from $\mathrm{AM}$ with dioxan as the polymerizing medium with lithium metal as the initiator. Formation of PAM from AM with lithium metal unequivocally demonstrates the free-radical nature of initiation of the polymerization reaction along with the usual anionic mechanism [as evidenced by the presence of characteristic PBAL ${ }^{1} \mathrm{H}$ NMR peaks in Fig. 2(c)].

In short, the ESR experiments with the lithium-initiated AN system and ${ }^{1} \mathrm{H}$ NMR spectra of the product obtained from lithium-initiated AM in dioxan strongly point to the dual nature-of both anion radical and free radical-lithium metal as an initiator in vinyl polymerization.

\section{References}

1. F. E. Matthews and E. H. Strange, Brit. Pat. 24,790 (1910).

2. C. Harries, Annalen Der Chemie 383, 157 (1911).

3. C. Walling, E. R. Briggs, W. Cummings, and F. R. Mayo, J. Am. Chem. Soc., 72, 48 (1950).

4. F. W. Stavely et al., Ind. Eng. Chem., 48, 778 (1956).

5. K. F. O'Driscoll, R. J. Boudreau, and A. V. Tobolsky, J. Polym. Sci., 31, 115 (1958).

6. K. F. O'Driscoll and A. V. Tobolsky, J. Polym. Sci., (a) 31, 123 (1958); (b) 37, 363 (1959).

7. A. V. Tobolsky and P. B. Hartley, J. Polym. Sci. A-1, 1, 15 (1963).

8. C. G. Overberger and N. Yamamoto, J. Polym. Sci. Lett., 3, 569 (1965). 
9. M. Szwarc, Makromol. Chem., 35, 132 (1960).

10. D. S. Breslow, G. E. Hulse, and A. S. Matlack, J. Am. Chem. Soc., 79, 3760 (1957).

11. M. Asai, M. Tsuboi, T. Shimanouchi, and S. Mizushima, J. Phys. Chem., 59, 322 (1955).

12. K. Yamaguchi and Y. Minoura, J. Polym. Sci. A-1, 10, 1217 (1910).

13. F. A. Bovey and G. V. D. Tiers, J. Polym. Sci. A-1, 1, 849 (1963).

14. V. R. Pai Vernekar and B. Shaha, Polym. Commun., 25, 363 (1984).

15. J. D. Glickson and J. Applequist, Macromolecules, 2, 628 (1969).

16. C. H. Bamford and A. D. Jenkins, Proc. R. Soc. London, A216, 515 (1953).

17. V. R. Pai Vernekar and R. Vasanthakumari, J. Solid State Chem., 46, 2682(1982).

18. C. H. Bamford, A. D. Jenkins, M. C. R. Symons, and M. G. Townsend, J. Polym. Sci., 34, 181 (1959).

19. Y. Tabata, in Adv. Macromol. Chem., 1, 283 (1968).

Received April 8, 1985

Accepted July 12, 1985 